\title{
Mild postoperative acute kidney injury and outcomes after surgery for congenital heart disease
}

\author{
Marnie L. Taylor, MD, ${ }^{\mathrm{a}}$ Fabio Carmona, MD, ${ }^{\mathrm{a}}$ Ravi R. Thiagarajan, MBBS, MPH, ${ }^{\mathrm{a}, \mathrm{d}}$ Lauren Westgate, RN, \\ CPNP, ${ }^{\mathrm{a}}$ Michael A. Ferguson, MD, ${ }^{\mathrm{b}, \mathrm{d}}$ Pedro J. del Nido, MD, ${ }^{\mathrm{c}, \mathrm{d}}$ and Satish K. Rajagopal, MD ${ }^{\mathrm{a}, \mathrm{d}}$
}

Objective: The effect of mild acute kidney injury (AKI) on outcomes after heart surgery in children is unclear. We sought to characterize the epidemiology of mild AKI associated with surgery for congenital heart disease (CHS-AKI) in children.

\begin{abstract}
Methods: We conducted a single-center, retrospective cohort study of 693 patients (aged 6 days-18 years) who underwent heart surgery in 2009. The prevalence of AKI within 72 hours of surgery was determined using the 3 -stage Acute Kidney Injury Network criteria. Factors associated with both hospital length of stay and AKI were used in a proportional hazards model to test the association of stage 1 AKI with hospital length of stay.
\end{abstract}

Results: The median age of the patients was 11.5 months (interquartile range, 3-54 months). Eighteen percent of the cohort had single ventricle heart disease and 54\% underwent RACHS- 1 category 3 or higher surgery. The prevalence of stages 1,2 , and 3 AKI in this cohort was $11 \%(n=77), 3 \%(n=19)$, and $1 \%(n=8)$, respectively. Factors independently associated with AKI were prematurity, single ventricle physiology, peak postoperative lactic acid concentration, cardiopulmonary bypass time, and a history of heart surgery. Stage 2 or greater CHS-AKI was associated with hospital length of stay (adjusted hazard ratio [AHR], $0.53 ; 95 \%$ confidence interval $[\mathrm{CI}], 0.33-0.87 ; P=.01$ ), but stage 1 was not (AHR, 0.85; 95\% CI, 0.66-1.10; $P=.22$ ).

Conclusions: AKI occurs after surgery for congenital heart disease but may be less common than previously reported. Although moderate to severe CHS-AKI is independently associated with prolonged recovery after heart surgery, mild disease does not appear to be. (J Thorac Cardiovasc Surg 2013;146:146-52)

Data suggesting adverse clinical outcomes for heterogeneous populations of critically ill patients with acute kidney injury (AKI) are accumulating. ${ }^{1-4}$ Mild AKI after cardiac surgery in adults (a serum creatinine increase of 0.3 $\mathrm{mg} / \mathrm{dL}$ from baseline) occurs in up to $30 \%$ of patients and is associated with increased rates of morbidity and mortality, increased health care resource utilization, and longer lengths of stay in the hospital. ${ }^{5-7}$ The few epidemiologic studies of AKI in children undergoing surgery for congenital heart disease report an association with adverse outcomes and an overall prevalence of $36 \%$ to $52 \%$, the majority $(57 \%-61 \%)$ of which is limited to mild disease. These studies were limited by sample sizes that precluded assessment of the effects of mild AKI on outcomes and, in some instances, suboptimal control for potentially important confounders of this complex relationship. ${ }^{8-11}$ Extrapolating from data in adults and

From the Departments of Cardiology, ${ }^{\mathrm{a}}$ Medicine, ${ }^{\mathrm{b}}$ and Cardiac Surgery, ${ }^{\mathrm{c}}$ Children's Hospital Boston; and Harvard Medical School, ${ }^{\mathrm{d}}$ Boston, Mass.

Funded by the Rochelle Rose Cardiac Intensive Care Unit Clinical Research Fund. Disclosures: Authors have nothing to disclose with regard to commercial support.

M.L.T. and F.C. contributed equally to this work.

Received for publication June 20, 2012; revisions received Aug 15, 2012; accepted for publication Sept 12, 2012; available ahead of print Oct 5, 2012.

Address for reprints: Satish K. Rajagopal, MD, Department of Cardiology, Children's

Hospital Boston, 300 Longwood Ave, Boston, MA 02115 (E-mail: Satish.

Rajagopal@cardio.chboston.org).

$0022-5223 / \$ 36.00$

Copyright (c) 2013 by The American Association for Thoracic Surgery

http://dx.doi.org/10.1016/j.jtcvs.2012.09.008 from the few available epidemiologic studies of AKI associated with surgery for congenital heart disease (CHS-AKI) in children, investigators have begun to propose and conduct a variety of prospective trials targeting the amelioration of CHS-AKI with the hope of improving outcomes. ${ }^{12-14}$ Given the paucity of existing data, the limitations of the previous studies, and the accelerating interest in attempting to decrease the frequency and severity of CHS-AKI, we undertook this study for 4 principal reasons: (1) to determine the prevalence and severity of CHS-AKI, (2) to determine which factors are associated with the development of CHS-AKI, (3) to determine whether mild AKI is associated with adverse clinical outcomes, and (4) to determine what proportion of patients with CHS-AKI have renal dysfunction at the time of hospital discharge. We hypothesized that mild CHS-AKI would not be associated with prolonged recovery from heart surgery after controlling for confounders.

\section{MATERIALS AND METHODS Setting and Patients}

This retrospective cohort study was conducted in the 29-bed Cardiac Intensive Care Unit at Children's Hospital Boston. We included all patients who (1) underwent Risk Adjustment for Congenital Heart Surgery-1 (RACHS-1) ${ }^{15}$ classifiable cardiac surgery at our institution during the 2009 calendar year, (2) were more than 5 days of age and less than 18 years of age on the day of surgery, and (3) were free of renal replacement therapy at the time of surgery. For patients who underwent more than 1 operation during the study period, each operative procedure was handled as a separate 


\section{Abbreviations and Acronyms \\ AHR = adjusted hazard ratio \\ AKI $=$ acute kidney injury \\ AKIN = Acute Kidney Injury Network \\ CHS-AKI = acute kidney injury associated with \\ surgery for congenital heart disease \\ $\mathrm{CI}=$ confidence interval \\ pRIFLE $=$ pediatric modification of RIFLE classification system \\ RACHS-1 = Risk Adjustment for Congenital Heart Surgery-1 \\ RIFLE = risk, injury, failure, loss of kidney function, and end-stage renal failure}

event provided the patient was discharged home between operations. The Committee on Clinical Investigation at Children's Hospital Boston approved the study and waived the requirement for written informed consent.

\section{Definition of Variables and Outcomes}

The primary predictor variable for the study was the degree of AKI, defined using the Acute Kidney Injury Network's (AKIN) 3-tiered staging system. ${ }^{16}$ The definitions of the 3 stages of AKI in the in AKIN classification are as follows: stage 1, increase in plasma creatinine of $0.3 \mathrm{mg} / \mathrm{dL}$ or increase in plasma creatinine to $150 \%$ to $200 \%$ ( $>1.5$-fold to 2-fold increase) of baseline; stage 2, increase in plasma creatinine to $200 \%$ to $300 \%$ ( $>2$-fold to 3 -fold) of baseline; stage 3, increase in plasma creatinine to more than $300 \%$ ( $>3$-fold) of baseline (with a rise of at least $0.5 \mathrm{mg} / \mathrm{dL}$ ), or a postoperative plasma creatinine concentration $\geq 4.0 \mathrm{mg} /$ $\mathrm{dL}$, or need for postoperative renal replacement therapy. Stages 2 and 3 AKI were grouped together a priori inasmuch as the prevalence of stage $3 \mathrm{AKI}$ is low in our institution. Recognizing the error inherent in creatinine measurements and the low creatinine concentrations in most children, we required a minimum increase in plasma creatinine of $0.2 \mathrm{mg} / \mathrm{dL}$ to meet criteria for AKI. Justifying this cutoff, the coefficient of variation for the Roche mass spectroscopy-traceable enzymatic creatinine assay performed in the Children's Hospital Boston chemistry laboratory is $1.77 \%$.

Given the lack of consensus regarding the most appropriate definition of AKI in children, the pediatric modification of the RIFLE classification system (risk, injury, failure, loss of kidney function, and end-stage renal failure) was also used to categorize patients into 1 of the 4 pRIFLE AKI stages; none, risk, injury, or failure. ${ }^{10}$ The primary difference between the AKIN and pRIFLE staging systems is the use of a change in estimated creatinine clearance to categorize patients in the latter, whereas the former relies solely on the fold change in creatinine concentration. This definition difference results in a greater number of children being categorized as having mild AKI (risk) in the pRIFLE staging system than in the AKIN system. ${ }^{17}$

Blood for the creatinine recorded as the "baseline" value was drawn within 5 days of surgery. Each patient's daily morning creatinine value was recorded for postoperative days 0 to 3 . The creatinine value obtained closest to hospital discharge, if outside the 72-hour postoperative window, was recorded as the "discharge creatinine." The updated Schwartz formula $(0.413 \cdot$ length $[\mathrm{cm}] /$ creatinine $[\mathrm{mg} / \mathrm{dL}])$ was used to calculate the estimated creatinine clearance. ${ }^{18}$ The following cutoffs (corresponding to $50 \%$ of normal mean glomerular filtration rate for age) were used to determine the presence or absence of renal dysfunction before surgery and at the time of discharge: 6 to 13 days of age $\left(21 \mathrm{~mL} \cdot \mathrm{min}^{-1}\right.$. $\left.1.73 \mathrm{~m}^{-2}\right), 14$ to 55 days of age $\left(33 \mathrm{~mL} \cdot \mathrm{min}^{-1} \cdot 1.73 \mathrm{~m}^{-2}\right), 56$ days to
2 years of age $\left(48 \mathrm{~mL} \cdot \min ^{-1} \cdot 1.73 \mathrm{~m}^{-2}\right)$, and 2 to 18 years of age $\left(60 \mathrm{~mL} \cdot \min ^{-1} \cdot 1.73 \mathrm{~m}^{-2}\right){ }^{19}$

Health care-associated infections, defined using definitions put forth by the Centers for Disease Control, included ventilator-associated pneumonia, endotracheal tube- associated tracheitis, urinary tract infection, catheterassociated bloodstream infections, and superficial and deep sternal wound infections. Infants less than 3 months of age at the time of surgery were categorized as premature if they were born at less than 38 weeks' gestation. Central nervous system injury was defined as seizure activity (electrographic or clinical) or radiologic evidence of ischemia and/or hemorrhage by ultrasound, computed tomography, and/or magnetic resonance imaging. Congenital anomalies included syndromes, known disease causing chromosomal abnormalities, and/or major noncardiac structural defects (eg, cleft palate, solitary kidney, esophageal atresia, and so on); minor structural defects were not included (eg, skin tags, superficial sacral dimple). Preoperative mechanical ventilation was defined as the need for a period of mechanical ventilation during the same hospitalization, but before surgery. Patients categorized as having single ventricle physiology included all patients whose anatomy precluded biventricular repair at the time of surgery. Necrotizing enterocolitis was diagnosed by either the intensive care unit attending physician or a general surgery consultant using clinical judgment and radiography. Diaphragm paresis or paralysis was diagnosed by ultrasound and/or fluoroscopy. Chylothorax was deemed to be present by pleural fluid analysis and a switch to a low-fat formula. Heart block was defined as documented atrioventricular node disturbance necessitating temporary or permanent ventricular pacing. Arrhythmia was defined as the presence of any abnormal rhythm necessitating intervention (pharmacologic and/or pacing). Noninvasive ventilation techniques included bilevel positive airway pressure, continuous positive airway pressure, and/or high-flow nasal cannula.

Unintended reexploration was defined as any procedure intended to control bleeding and/or relieve tamponade. Repeat surgery was defined as any unintended cardiac surgery, including shunt manipulation but excluding delayed sternal closure and reexploration. Designation as a planned or emergency surgery (eg, repair of obstructed total anomalous pulmonary venous drainage) was made by the attending cardiothoracic surgeon at the time of surgery. Cachexia and obesity were defined as a weight-for-age Z-score at the time of surgery of 2 or less or more than 2, respectively. Preoperative polycythemia and anemia were defined by hemoglobin concentrations outside the normal range for age. Peak lactate was the highest postoperative lactate within 24 hours of surgery. Each index surgery was assigned a RACHS-1 category. For patients who underwent more than 1 discrete surgical intervention during the same operation (eg, mitral valvuloplasty and subaortic membrane resection), the highest RACHS-1 category among the individual components was assigned to the procedure.

The primary outcome variable was postoperative hospital length of stay. Patients who died before hospital discharge were included in the nonparametric analyses by assigning their hospital length of stay to the maximal value for those surviving to discharge. When patients had more than $1 \mathrm{car}-$ diac surgical procedure during the same hospitalization, the day of the first operation was used to calculate all postoperative length of stay parameters.

\section{Statistical Analysis}

Predictive Analytics Software version 18.0.0 (SPSS, Inc, Chicago, Ill) and Stata 11 (StataCorp, College Station, Tex) were used for statistical analysis. Data are reported as frequency (n) with proportion (percent) or median value with interquartile range (25th-75th percentile). The Wilcoxon rank sum and Kruskal-Wallis tests were used to test the statistical significance of continuous data. The $\chi^{2}$ test and the $\chi^{2}$ test for trend were used to test the statistical significance of categorical data, and Fisher's exact test was used when more than $20 \%$ of the cells had expected counts of less than 5. Multivariable logistic regression was used to identify preoperative, intraoperative, and early postoperative factors associated with AKI using covariates with a $P$ value of .1 or less on bivariate statistical testing. 
Given the anticipated low prevalence of severe AKI in this cohort, we made the a priori decision to group moderate and severe AKI (stages 2 and 3) together for statistical analysis. To maintain model stability, we added the RACHS- 1 category into the logistic regression model as a dichotomous variable (RACHS- 1 category 3 and RACHS- 1 category $<3$ ), dividing the cohort into roughly equal-size groups.

Factors associated with AKI and hospital length of stay were identified by bivariate analyses and subsequently included in a Cox proportional hazards regression model of the primary outcome if the $P$ value was .1 or less. No selection procedure was used for entry of the candidate variables into the models. The proportional hazards modeling was repeated after substituting the pRIFLE AKI stages for the AKIN AKI stages to determine whether the effect of AKI on outcomes differed by AKI staging system. The criterion for variable inclusion in the final models was set at $P \leq$ .05. Candidate variables in the Cox proportional hazards model were assessed for time dependency by assessing whether log minus log survival plots were parallel. Given that the number of postoperative days to hospital discharge (ie, postoperative hospital length of stay) was the primary outcome modeled, a hazard ratio of less than 1 signifies a lower instantaneous risk of hospital discharge at any given time point or, more intuitively, a greater risk of remaining hospitalized.

\section{RESULTS}

\section{Study Population, Change in Plasma Creatinine Concentration, and Prevalence of AKI}

The initial study cohort comprised 721 patients. Twentyeight patients were subsequently excluded (missing baseline creatinine $[\mathrm{n}=18]$, preoperative extracorporeal membrane oxygenation [n=1], and extracorporeal membrane oxygenation within 72 hours of surgery $[n=9])$, leaving 693 patients representing the primary study cohort. The median age of the children in the study at the time of surgery was 11.5 months (interquartile range, 3.2-54 months). Of these children, $18 \%(\mathrm{n}=121)$ had single ventricle heart disease, $42 \%(n=289)$ had previously undergone surgery for congenital heart disease, $35 \%(\mathrm{n}=240)$ were admitted to the hospital before surgery, and $12 \%(n=82)$ were supported with a ventilator in the period before their index heart surgery. The distribution of the operations performed in this series by RACHS- 1 categories 1 to 6 was as follows: $10 \%$ $(\mathrm{n}=67), 37 \%(\mathrm{n}=257), 40 \%(\mathrm{n}=276), 11 \%(\mathrm{n}=75)$, $0 \%(n=0)$, and $3 \%(n=18)$, respectively (Table 1$)$. Ten patients $(1.4 \%)$ had renal dysfunction before surgery (an estimated creatinine clearance of $\leq 50 \%$ predicted for age), the etiology of which was not documented. Criteria for stages 1 (mild), 2 (moderate), and 3 (severe) AKI were met within 72 hours of surgery in $11 \%(n=77), 3 \%$ $(\mathrm{n}=19)$, and $1 \%(\mathrm{n}=8)$ of the 693 patients, respectively, for an overall prevalence of $15 \%$. Absolute plasma creatinine concentrations stratified by age and development of AKI are presented in Table 2. Six patients required renal replacement therapy in the postoperative period $(1 \%)$, but none was within the 72-hour postoperative window.

\section{Factors Associated With the Development of AKI}

Important preoperative, intraoperative, and early postoperative factors were tested for an association with
AKI (Table 1). There was no detectable difference in preoperative renal function between those in whom AKI developed and those in whom it did not develop. Multivariable logistic regression modeling of AKI after cardiac surgery demonstrated independent associations with prematurity, single ventricle physiology, peak 24-hour postoperative lactic acid concentration, duration of cardiopulmonary bypass, and history of heart surgery, but not with higher RACHS-1 category, presence of residual heart lesions (evidenced by need for postoperative surgical or catheter-based reintervention), need for delayed sternal closure, nor presence of preoperative polycythemia (Table 3).

\section{Outcomes of Patients With Postoperative AKI}

Postoperative AKI, particularly stage 2 or 3 disease, was associated with increased postoperative morbidity and mortality on univariate analysis (Table 1). Those with postoperative AKI were more likely to die after surgery and had longer intensive care unit and hospital lengths of stay than those without AKI. As shown in Table 4, multivariable proportional hazards modeling demonstrated that whereas moderate to severe AKI (stages 2 and 3) was independently associated with hospital length of stay (adjusted hazard ratio [AHR], 0.53; 95\% confidence intervals [CI], 0.33-0.87; $P=.01$ ), mild AKI (stage 1) was not (AHR, $0.85 ; 95 \% \mathrm{CI}, 0.66-1.10 ; P=.22$ ). The same relationships of AKI stage to length of stay were maintained after substituting the pRIFLE stage for AKIN stage; risk level AKI (AHR, 1.01; 95\% CI, $0.84-1.23 ; P=.88$ ) and injury and failure level AKI (AHR, 0.60; 95\% CI, 0.37-0.98; $P=.04$ ). With respect to renal outcomes, children who had AKI develop in the early postoperative period were more likely to require a limited period of renal replacement therapy in the postoperative period than those who did not $(4 \%$ vs $0.3 \% ; P=.006)$ but were no more likely to be discharged with renal dysfunction $(2 \%$ vs $1 \% ; P=.3)$.

\section{DISCUSSION}

In this study, we found a $15 \%$ prevalence of CHS-AKI in a single-center cohort of nearly 700 infants, children, and young adults who underwent a wide variety of cardiac surgical procedures. The majority of patients in whom AKI developed $(74 \%)$ were limited to mild disease (stage 1), while $18 \%$ and $8 \%$, respectively, met stage 2 and 3 criteria. Multivariable modeling demonstrated that prematurity, single ventricle physiology, a history of cardiac surgery, peak postoperative lactic acid concentration, and duration of cardiopulmonary bypass were associated with the development of CHS-AKI, whereas residual heart disease and higher RACHS-1 category were not. After controlling for a variety of potential confounders, children with more severe CHS-AKI (stage 2 or 3 ) were $35 \%$ more likely to remain 
TABLE 1. Perioperative characteristics and outcomes of the study cohort

\begin{tabular}{|c|c|c|c|c|}
\hline & No AKI $(\mathbf{n}=\mathbf{5 8 9})$ & Stage 1 AKI $(n=77)$ & Stages 2 and 3 AKI $(n=27)$ & $P$ value \\
\hline \multicolumn{5}{|l|}{ Preoperative variables } \\
\hline Age at surgery (mo) & $11.4(3.3-58.3)$ & $12.3(3.0-44.6)$ & $9.0(3.0-30.8)$ & .50 \\
\hline Length at surgery $(\mathrm{cm})^{*}$ & $73(59-104)$ & $74(57-94)$ & $68(56-87)$ & .38 \\
\hline Weight at surgery $(\mathrm{kg})$ & $8.3(4.72-17.5)$ & $8.8(4.4-13.7)$ & $7.8(3.7-12.6)$ & .39 \\
\hline Gender (female) & $282(48)$ & $35(46)$ & $9(33)$ & .32 \\
\hline Premature birth & $28(5)$ & $9(12)$ & $2(7)$ & .04 \\
\hline Preoperative mechanical ventilation & $66(11)$ & $10(13)$ & $6(22)$ & .21 \\
\hline Preoperative hospital admission & $199(34)$ & $30(39)$ & $11(41)$ & .53 \\
\hline Single ventricle physiology & $83(14)$ & $25(33)$ & $13(48)$ & $<.001$ \\
\hline Previous heart surgery & $227(39)$ & $44(57)$ & $18(67)$ & $<.001$ \\
\hline Genetic syndrome & $143(24)$ & $20(26)$ & $3(11)$ & .27 \\
\hline Cachectic & $154(26)$ & $23(30)$ & $7(26)$ & .78 \\
\hline Obesity & $7(1)$ & $0(0)$ & $1(4)$ & .30 \\
\hline Anemia & $59(10)$ & $10(13)$ & $1(4)$ & .38 \\
\hline Polycythemia & $223(38)$ & $40(52)$ & $18(67)$ & .001 \\
\hline Creatinine (mg/dL) & $0.3(0.3-0.4)$ & $0.3(0.3-0.4)$ & $0.3(0.2-0.4)$ & .14 \\
\hline Estimated creatinine clearance $\left(\mathrm{mL} \cdot \mathrm{min}^{-1} \cdot 1.73 \mathrm{~m}^{-2}\right)^{*}$ & $107(80-131)$ & $97(73-128)$ & $128(81-169)$ & .18 \\
\hline Kidney disease* & $8(1)$ & $1(1)$ & $1(4)$ & .64 \\
\hline \multicolumn{5}{|l|}{ Operative variables } \\
\hline RACHS-1 category & & & & $<.001$ \\
\hline 1 & $67(11)$ & $0(0)$ & $0(0)$ & \\
\hline 2 & $232(39)$ & $21(27)$ & $4(15)$ & \\
\hline 3 & $218(37)$ & $45(58)$ & $13(48)$ & \\
\hline 4 & $59(10)$ & $8(10)$ & $8(30)$ & \\
\hline 6 & $13(2)$ & $3(4)$ & $2(7)$ & \\
\hline Emergency surgery & $6(1)$ & $1(1)$ & $1(4)$ & .44 \\
\hline Planned surgery & $575(98)$ & $74(96)$ & $26(96)$ & .69 \\
\hline CPB time (min) & $91(60-131)$ & $128(88-173)$ & $138(92-227)$ & $<.001$ \\
\hline Aortic crossclamp time (min) & $54(25-76)$ & $75(33-101)$ & $64(0-118)$ & .003 \\
\hline Delayed sternal closure & $50(9)$ & $15(20)$ & $10(37)$ & $<.001$ \\
\hline Circulatory arrest used & $64(11)$ & $7(9)$ & $3(11)$ & .89 \\
\hline \multicolumn{5}{|l|}{ Postoperative variables } \\
\hline Peak lactate $(\mathrm{mmol} / \mathrm{L})$ & $2.1(1.6-2.9)$ & $3.2(2.1-4.7)$ & $5.7(3.9-7.5)$ & $<.001$ \\
\hline Heart block requiring temporary pacing & $23(4)$ & $11(14)$ & $3(11)$ & $<.001$ \\
\hline Arrhythmia & $86(15)$ & $22(29)$ & $13(48)$ & $<.001$ \\
\hline Necrotizing enterocolitis & $6(1)$ & $1(1)$ & $4(15)$ & $<.001$ \\
\hline Diaphragm paresis & $6(1)$ & $2(3)$ & $3(11)$ & $<.001$ \\
\hline Chylothorax & $13(2)$ & $6(8)$ & $5(19)$ & $<.001$ \\
\hline Arrest requiring $\mathrm{CPR}$ & $3(0.5)$ & $3(4)$ & $0(0)$ & .009 \\
\hline Health care-associated infection & $32(5)$ & $8(10)$ & $6(22)$ & .001 \\
\hline Need for blood transfusion & $255(43)$ & $50(65)$ & $23(85)$ & $<.001$ \\
\hline Total packed RBCs transfused (mL/kg) & $0(0-14)$ & $14(0-20)$ & $24(14-45)$ & $<.001$ \\
\hline RBCs transfused on POD $0(\mathrm{~mL} / \mathrm{kg})$ & $0(0-10)$ & $7(0-15)$ & $15(4-30)$ & $<.001$ \\
\hline Red blood cells transfused on POD $1(\mathrm{~mL} / \mathrm{kg})$ & $0(0-0)$ & $0(0-9)$ & $0(0-14)$ & $<.001$ \\
\hline Red blood cells transfused on POD $2(\mathrm{~mL} / \mathrm{kg})$ & $0(0-0)$ & $0(0-0)$ & $0(0-15)$ & $<.001$ \\
\hline Tracheostomy & $3(.5)$ & $2(2.6)$ & $0(0)$ & .11 \\
\hline Noninvasive ventilation & $81(14)$ & $17(22)$ & $12(44)$ & $<.001$ \\
\hline Central nervous system injury & $13(2)$ & $5(7)$ & $4(15)$ & $<.001$ \\
\hline Reintubation & $30(5)$ & $6(8)$ & $4(15)$ & .08 \\
\hline Unanticipated procedure (surgery or catheter) $\dagger$ & $47(8)$ & $8(10)$ & $17(63)$ & $<.001$ \\
\hline Mediastinal reexploration & $15(3)$ & $6(8)$ & $7(26)$ & $<.001$ \\
\hline Extracorporeal membrane oxygenation use & $8(1)$ & $2(3)$ & $3(11)$ & .001 \\
\hline
\end{tabular}


TABLE 1. Continued

\begin{tabular}{|c|c|c|c|c|}
\hline & No AKI $(n=589)$ & Stage 1 AKI $(n=77)$ & Stages 2 and 3 AKI $(n=27)$ & $P$ value \\
\hline \multicolumn{5}{|l|}{ Outcomes } \\
\hline Renal replacement therapy & $2(.3)$ & $1(1)$ & $3(11)$ & $<.001$ \\
\hline ICU length of stay (d) & $2(1-4)$ & $4(2-8)$ & $11(6-17)$ & $<.001$ \\
\hline ICU readmission & $28(5)$ & $5(7)$ & $9(33)$ & $<.001$ \\
\hline Hospital length of stay (d) & $6(4-10)$ & $9(7-21)$ & $33(19-41)$ & $<.001$ \\
\hline Death before discharge & $5(.8)$ & $1(1)$ & $6(22)$ & $<.001$ \\
\hline Creatinine at discharge $(\mathrm{mg} / \mathrm{dL}) \ddagger$ & $0.3(0.2-0.4)$ & $0.3(0.2-0.4)$ & $0.3(0.2-0.3)$ & .17 \\
\hline $\begin{array}{l}\text { Estimated creatinine clearance at discharge } \\
\quad\left(\mathrm{mL} \cdot \min ^{-1} \cdot 1.73 \mathrm{~m}^{-2}\right) \S\end{array}$ & $113(82-134)$ & $97(75-122)$ & $107(86-121)$ & .18 \\
\hline Kidney disease at discharge $\S$ & $4(1)$ & $2(3)$ & $0(0)$ & .38 \\
\hline
\end{tabular}

hospitalized at any given time than were those whose renal function did not meet AKI criteria (AHR, 0.53; 95\% CI, 0.33-0.87), but there was no detectable difference in duration of hospitalization between those in whom only mild disease developed and those whose renal function did not meet AKI criteria. Postoperative AKI was not associated with renal dysfunction at hospital discharge, although the overall prevalence of the latter was low $(1 \%)$.

This study extends previous work describing the epidemiology of CHS-AKI in children. ${ }^{8-13}$ The prevalence of AKI was lower in the current study than in those previously published $(15 \%$ vs $36 \%-59 \%)$. There are several possible explanations for this discrepancy. Subtle variations in the AKI definitions used in the previous studies might have led to higher prevalence estimates, that is, pRIFLE without restriction to a specified postoperative window in Zappitelli and associates, ${ }^{9}$ AKIN criteria within 7 days of surgery (vs 3 days) in $\mathrm{Li}$ and colleagues, ${ }^{11}$ and AKIN criteria with urine output criteria in Blinder and coworkers. ${ }^{8}$ Differing patient demographics may have contributed to the discrepant findings. This is most apparent in the study by Blinder and colleagues, ${ }^{8}$ who restricted their study to infants who are at higher risk for a variety of adverse outcomes, including AKI. Era effect, related to improvements in perioperative management stemming from a heightened awareness of the potentially important consequences of AKI, may also be contributing to the differences inasmuch as the majority of children in the previous studies underwent surgery in a 6-year period preceding the current study.

Despite the differences in overall prevalence, all 4 studies demonstrated that AKI was limited to mild disease in the majority of those affected (57\%-77\%). Each study attempted to identify risk factors for the development of CHS-AKI. Strengthening their findings, we corroborated the independent association between CHS-AKI and longer cardiopulmonary bypass times and the lack of an association between CHS-AKI and surgical complexity (RACHS-1 category). Several other new potential CHS-AKI risk factors were identified in the present study, including single ventricle physiology, prematurity, peak 24-hour postoperative lactate concentration, and previous heart surgery.

The effects of mild AKI on clinical outcomes after surgery for congenital heart disease have not been studied adequately, despite repeated observations that the majority of AKI remains mild. Our finding that mild CHS-AKI is not associated with hospital length of stay corroborates those of Blinder and associates ${ }^{8}$ in their study of infants undergoing heart surgery, but it contradicts several studies of adults undergoing heart surgery. The reasons underlying the apparent difference in epidemiology of mild AKI after heart surgery between adults and children are not clear, but several possible explanations warrant consideration. Renal recovery potential after acute insults such as cardiopulmonary bypass may be more robust in children, rendering mild CHS-AKI

TABLE 2. Creatinine concentrations stratified by age at surgery and development of AKI

\begin{tabular}{|c|c|c|c|c|c|c|}
\hline \multirow[b]{2}{*}{ Variable } & \multicolumn{2}{|c|}{$6 \mathrm{~d}-2 \mathrm{y}$} & \multicolumn{2}{|c|}{$2-10 y$} & \multicolumn{2}{|c|}{$10-18 y$} \\
\hline & AKI & No AKI & AKI & No AKI & AKI & No AKI \\
\hline Preop creatinine (mg/dL) & $0.3(0.2-0.4)$ & $0.3(0.2-0.4)$ & $0.3(0.3-0.3)$ & $0.3(0.3-0.4)$ & $0.6(0.4-0.8)$ & $0.6(0.5-0.7)$ \\
\hline Postop max creatinine $(\mathrm{mg} / \mathrm{dL})$ & $0.6(0.5-0.7)$ & $0.3(0.3-0.4)$ & $0.6(0.5-0.6)$ & $0.4(0.3-0.4)$ & $1.2(1.0-1.5)$ & $0.7(0.6-0.8)$ \\
\hline Max creatinine change $(\mathrm{mg} / \mathrm{dL})$ & $0.2(0.2-0.4)$ & $0.0(0.0-0.1)$ & $0.3(0.2-0.4)$ & $0.0(0.0-0.1)$ & $0.5(0.4-0.5)$ & $0.0(0.0-0.1)$ \\
\hline
\end{tabular}

Values are expressed as median (interquartile range). AKI, Acute kidney injury; Preop, preoperative; max, maximum; Postop, Postoperative. 
TABLE 3. Logistic regression analysis of AKI after surgery for congenital heart disease $(n=693)$

\begin{tabular}{lccc}
\hline \multicolumn{1}{c}{ Variable } & Adjusted OR & $\mathbf{9 5} \%$ CI & $\boldsymbol{P}$ value \\
\hline Premature birth & 2.4 & $1.01-5.8$ & .05 \\
Single ventricle physiology & 1.8 & $1.01-3.1$ & .05 \\
Peak postoperative lactate & 1.3 & $1.01-1.5$ & $<.001$ \\
$\quad$ concentration (per 1 mmol/L) & & & \\
RACHS-1 category $\geq 3$ & 1.5 & $0.8-2.6$ & .18 \\
CPB time (per min) & 1.005 & $1.00-1.009$ & .04 \\
Need for postop intervention & 1.7 & $0.78-3.52$ & .19 \\
Delayed sternal closure & 0.7 & $0.3-1.7$ & .46 \\
Postop blood transfusion quantity & 1.010 & $0.998-1.023$ & .10 \\
$\quad$ per 1 mL/kg) & & & \\
Preop polycythemia & 1.2 & $0.7-2.0$ & .56 \\
Previous heart surgery & 1.9 & $1.1-3.3$ & .03 \\
\hline
\end{tabular}

$A K I$, Acute kidney injury; $O R$, odds ratio; $C I$, confidence interval; $R A C H S$, Risk Adjustment for Congenital Heart Surgery; $C P B$, cardiopulmonary bypass; postop, postoperative; preop, preoperative.

irrelevant with respect to outcomes. ${ }^{3,20,21}$ This possibility is bolstered by our observation that those children in whom CHS-AKI developed were no more likely to be discharged from the hospital with renal dysfunction that those who did meet AKI criteria. Alternatively, the existing definitions of AKI may be inadequate to accurately define a biologically relevant deterioration in renal function in children. 16,19 "Normal" serum creatinine ranges in children (with the exception of newborns) are lower than in adults, related to a higher glomerular filtration rate. The combination of lower baseline serum creatinine values, a definition of AKI based on percentage change in creatinine, and the inherent analytical error in creatinine testing may lead to misclassification of children without biologically significant kidney injury as having stage 1 AKI, thereby suppressing the effect of real, but mild AKI on outcomes. ${ }^{22}$

Several important limitations should be considered when interpreting the results of this study. First, there is the possibility that patient selection bias, related to the exclusion of patients secondary to missing data, affected the results of the study. However the number of such patients was small. Second, retrospective studies alone cannot prove causality, that is, moderate to severe AKI may be associated with, but not cause, prolongation of hospital length of stay. Third, the updated Schwartz formula was used to calculate estimated creatinine clearance and determine the presence of renal dysfunction before surgery and at discharge. Although the Schwartz formula is known to overestimate the glomerular filtration rate, for reasons of practicality and general consensus, it remains the only common method for estimating renal function in children. Fourth, there is a possibility that confounders of the relationship between AKI and outcomes were not considered. Fifth, given the low mortality rate, we were unable to evaluate the association between CHS-AKI and death.
TABLE 4. Proportional hazards model of hospital length of stay: The independent effect of AKI $(n=693)$

\begin{tabular}{|c|c|c|c|}
\hline Variable & AHR* & $95 \% \mathrm{CI}$ & $P$ value \\
\hline Age at surgery (per mo) & 1.002 & $1.001-1.003$ & .006 \\
\hline Premature birth & 0.63 & $0.44-0.91$ & .01 \\
\hline Preop mechanical ventilation & 0.61 & $0.46-0.81$ & .001 \\
\hline CNS injury & 0.51 & $0.28-0.92$ & .03 \\
\hline Necrotizing enterocolitis & 0.36 & $0.16-0.78$ & .009 \\
\hline Diaphragm paresis & 1.16 & $0.59-2.26$ & .67 \\
\hline Chylothorax & 0.70 & $0.43-1.13$ & .14 \\
\hline Heart block & 0.65 & $0.45-0.94$ & .02 \\
\hline Arrhythmia & 0.76 & $0.61-0.95$ & .02 \\
\hline Single ventricle & 0.68 & $0.53-0.87$ & .002 \\
\hline Tracheostomy & 0.49 & $0.19-1.24$ & .13 \\
\hline Noninvasive ventilation & 0.63 & $0.49-0.82$ & .001 \\
\hline Reintubation & 0.69 & $0.47-1.03$ & .07 \\
\hline Postop catheterization or surgery & 0.46 & $0.34-0.63$ & $<.001$ \\
\hline Delayed sternal closure & 0.78 & $0.55-1.12$ & .18 \\
\hline Reexploration & 0.81 & $0.48-1.36$ & .42 \\
\hline Polycythemia & 1.02 & $0.86-1.21$ & .80 \\
\hline CPR & 2.10 & $0.85-5.21$ & .1 \\
\hline Health care-associated infection & 0.37 & $0.25-0.53$ & $<.001$ \\
\hline Peak lactate (per $1 \mathrm{mmol} / \mathrm{L}$ ) & 0.90 & $0.85-0.95$ & $<.001$ \\
\hline $\mathrm{CPB}$ time & 1.29 & $0.93-1.78$ & .12 \\
\hline RACHS- 1 category & & & $<.001$ \\
\hline Category 1 & 1.00 & (reference) & \\
\hline Category 2 & 0.40 & $0.30-0.53$ & $<.001$ \\
\hline Category 3 & 0.26 & $0.19-0.36$ & $<.001$ \\
\hline Category 4 & 0.24 & $0.16-0.35$ & $<.001$ \\
\hline Category 6 & 0.33 & $0.18-0.62$ & .001 \\
\hline ECMO & 0.93 & $0.43-2.03$ & .85 \\
\hline ICU readmission & 0.38 & $0.26-0.54$ & $<.001$ \\
\hline Blood transfusion & 0.68 & $0.57-0.81$ & $<.001$ \\
\hline AKI stage & & & .02 \\
\hline Absent & 1.00 & (reference) & \\
\hline Stage 1 & 0.85 & $0.66-1.10$ & .22 \\
\hline Stages 2 and 3 & 0.53 & $0.33-0.87$ & .01 \\
\hline
\end{tabular}

Values are expressed as median (interquartile range) or $\mathrm{n}(\%)$ as appropriate. $A K I$, Acute kidney injury; $A H R$, adjusted hazard ratio; $C I$, confidence interval; Preop, preoperative; $C N S$, central nervous system; Postop, postoperative; $C P R$, cardiopulmonary resuscitation; $C P B$, cardiopulmonary bypass; RACHS, Risk Adjustment for Congenital Heart Surgery; ECMO, extracorporeal membrane oxygenation $I C U$, intensive care unit. *A hazard ratio of less than 1 indicates a greater likelihood of remaining hospitalized on any given day.

The findings of this study suggest that CHS-AKI may not be as common as previously thought and that trials targeting reduction of CHS-AKI should focus on those who have, or are likely to have, moderate to severe disease, inasmuch as mild disease does not appear to have much of an effect on outcomes. Further research, preferably a large, prospective, multicenter study, will be necessary to determine which risk factors predispose children to moderate to severe CHS-AKI and to determine whether CHS-AKI is associated with other clinically relevant outcomes. An accurate risk prediction model of CHS-AKI, ideally incorporating diagnostic biomarker data, would then set an appropriate stage for therapeutic trials targeting amelioration of AKI 
with the goal of improving the outcomes of children undergoing surgery for congenital heart disease.

\section{References}

1. Schneider J, Khemani R, Grushkin C, Bart R. Serum creatinine as stratified in the RIFLE score for acute kidney injury is associated with mortality and length of stay for children in the pediatric intensive care unit. Crit Care Med. 2010;38: 933-9.

2. Alkandari O, Eddington KA, Hyder A, Gauvin F, Ducruet T, Gottesman R, et al. Acute kidney injury is an independent risk factor for pediatric intensive care unit mortality, longer length of stay and prolonged mechanical ventilation in critically ill children: a two-center retrospective cohort study. Crit Care. 2011;15: R146.

3. Rosner MH, Okusa MD. Acute kidney injury associated with cardiac surgery. Clin J Am Soc Nephrol. 2006;1:19-32.

4. Barrantes F, Tian J, Vazquez R, Amoateng-Adjepong Y, Manthous CA. Acute kidney injury criteria predict outcomes of critically ill patients. Crit Care Med. 2008;36:1397-403.

5. Karkouti K, Wijeysundera DN, Yau TM, Callum JL, Cheng DC, Crowther M, et al. Acute kidney injury after cardiac surgery: focus on modifiable risk factors. Circulation. 2009;119:495-502.

6. Lassnigg A, Schmidlin D, Mouhieddine M, Bachmann LM, Druml W, Bauer P, et al. Minimal changes of serum creatinine predict prognosis in patients after cardiothoracic surgery: a prospective cohort study. J Am Soc Nephrol. 2004;15: 1597-605.

7. Englberger L, Suri RM, Li Z, Casey ET, Daly RC, Dearani JA, et al. Clinical accuracy of RIFLE and Acute Kidney Injury Network (AKIN) criteria for acute kidney injury in patients undergoing cardiac surgery. Crit Care. 2011; 15:R16.

8. Blinder JJ, Goldstein SL, Lee VV, Baycroft A, Fraser CD, Nelson D, et al. Congenital heart surgery in infants: effects of acute kidney injury on outcomes. $J$ Thorac Cardiovasc Surg. 2012;143:368-74.

9. Zappitelli M, Bernier PL, Saczkowski RS, Tchervenkov CI, Gottesman R, Dancea A, et al. A small post-operative rise in serum creatinine predicts acute kidney injury in children undergoing cardiac surgery. Kidney Int. 2009;76: 885-92.
10. Akcan-Arikan A, Zappitelli M, Loftis LL, Washburn KK, Jefferson LS, Goldstein SL. Modified RIFLE criteria in critically ill children with acute kidney injury. Kidney Int. 2007;71:1028-35.

11. Li S, Krawczeski CD, Zappitelli M, Devarajan P, Thiessen-Philbrook H, Coca SG, et al. Incidence, risk factors, and outcomes of acute kidney injury after pediatric cardiac surgery: a prospective multicenter study. Crit Care Med. 2011; 39:1493-9.

12. Bennett M, Dent CL, Ma Q, Dastrala S, Grenier F, Workman R, et al. Urine NGAL predicts severity of acute kidney injury after cardiac surgery: a prospective study. Clin J Am Soc Nephrol. 2008;3:665-73.

13. Devarajan P, Krawczeski CD, Nguyen MT, Kathman T, Wang Z, Parikh CR. Proteomic identification of early biomarkers of acute kidney injury after cardiac surgery in children. Am J Kidney Dis. 2010;56:632-42.

14. Krawczeski CD, Woo JG, Wang Y, Bennett MR, Ma Q, Devarajan P. Neutrophil gelatinase-associated lipocalin concentrations predict development of acute kidney injury in neonates and children after cardiopulmonary bypass. $J$ Pediatr. 2011;158:1009-15.e1.

15. Jenkins KJ, Gauvreau K, Newburger JW, Spray TL, Moller JH, Iezzoni LI. Consensus-based method for risk adjustment for surgery for congenital heart disease. J Thorac Cardiovasc Surg. 2002;123:110-8.

16. Mehta RL, Kellum JA, Shah SV, Molitoris BA, Ronco C, Warnock DG, et al. Acute Kidney Injury Network: report of an initiative to improve outcomes in acute kidney injury. Crit Care. 2007;11:R31.

17. Zappitelli M, Parikh CR, Akcan-Arikan A, Washburn KK, Moffett BS, Goldstein SL. Ascertainment and epidemiology of acute kidney injury varies with definition interpretation. Clin J Am Soc Nephrol. 2008;3:948-54.

18. Schwartz GJ, Munoz A, Schneider MF, Mak RH, Kaskel F, Warady BA, et al. New equations to estimate GFR in children with CKD. J Am Soc Nephrol. 2009;20:629-37.

19. Hogg RJ, Furth S, Lemley KV, Portman R, Schwartz GJ, Coresh J, et al. National Kidney Foundation's Kidney Disease Outcomes Quality Initiative clinical practice guidelines for chronic kidney disease in children and adolescents: evaluation, classification, and stratification. Pediatrics. 2003;111(6 Pt 1):1416-21.

20. Andreoli SP. Acute renal failure in the newborn. Semin Perinatol. 2004;28: 112-23.

21. Andreoli SP. Acute kidney injury in children. Pediatr Nephrol. 2009;24:253-63.

22. Waikar SS, Bonventre JV. Creatinine kinetics and the definition of acute kidney injury. J Am Soc Nephrol. 2009;20:672-9. 